\title{
Mental resilience, perceived immune functioning, and health
}

\author{
Marith Van Schrojenstein \\ Lantman' \\ Marlou Mackus' \\ Leila S Otten' \\ Deborah de Kruijff' \\ Aurora JAE van de Loo ${ }^{1,2}$ \\ Aletta D Kraneveld ${ }^{1,2}$ \\ Johan Garssen ${ }^{1,3}$ \\ Joris C Verster ${ }^{1,2,4}$ \\ 'Division of Pharmacology, \\ Utrecht University, Utrecht, the \\ Netherlands; 'Institute for Risk \\ Assessment Sciences, Utrecht \\ University, Utrecht, the Netherlands; \\ ${ }^{3}$ Nutricia Research, Utrecht, the \\ Netherlands; ${ }^{4}$ Centre for Human \\ Psychopharmacology, Swinburne \\ University, Melbourne, Australia
}

Correspondence: Joris C Verster Division of Pharmacology, Utrecht University, Universiteitsweg 99, 3584 CG Utrecht, the Netherlands

Tel +3I 0302536909

$\mathrm{Fax}+310302537900$

Email j.c.verster@uu.nl
This article was published in the following Dove Press journal:

Journal of Multidisciplinary Healthcare

21 March 2017

Number of times this article has been viewed

Background: Mental resilience can be seen as a trait that enables an individual to recover from stress and to face the next stressor with optimism. People with resilient traits are considered to have a better mental and physical health. However, there are limited data available assessing the relationship between resilient individuals and their perspective of their health and immune status. Therefore, this study was conducted to examine the relationship between mental resilience, perceived health, and perceived immune status.

Methods: A total of 779 participants recruited at Utrecht University completed a questionnaire consisting of demographic characteristics, the brief resilience scale for the assessment of mental resilience, the immune function questionnaire (IFQ), and questions regarding their perceived health and immune status.

Results: When correcting for gender, age, height, weight, smoker status, amount of cigarettes smoked per week, alcohol consumption status, amount of drinks consumed per week, drug use, and frequency of past year drug use, mental resilience was significantly correlated with perceived health $(r=0.233, p=0.0001)$, perceived immune functioning $(r=0.124, p=0.002)$, and IFQ score $(r=-0.185, p=0.0001)$.

Conclusion: A significant, albeit modest, relationship was found between mental resilience and perceived immune functioning and health.

Keywords: mental resilience, immune functioning, health, vitality, quality of life

\section{Introduction}

Vitality is often viewed as a physical aspect of health, but mental resilience is equally important in the overall experience of good health and high quality of life. ${ }^{1}$ Mental resilience stands for one's capacity to recover from extremes of trauma and stress, and it reflects a union of factors that encourage positive adaptation despite exposure to adverse life experiences. These factors include individual protective factors (positive personal perspective), family protective factors (family support), and social protective factors (social integration of the individual). ${ }^{2} \mathrm{~A}$ positive attitude enables one to cope with adverse life events, resulting in increased self-esteem, confidence, and a sense of well-being.

The term resilience can be seen in three different directions: a trait, an outcome, and a process. ${ }^{3}$ Resilience as a trait is a personal characteristic, and it is thought that resilience as a trait protects the individual against the impact of an adverse life event. Resilience as an outcome is seen as a tool to help an individual recover from the impact of an adverse life event. Researchers who see resilience as a process believe that when an individual experiences an adverse life event, the process of adapting to 
and recovering from the impact of this adverse life event can be defined as resilience. These definitions state that mental resilience is a more or less person-specific constant rather than a variable that can change over time. Some believe that the process of adapting to and recovering from the impact of an adverse life event leads to behavioral immunization or behavioral resilience. ${ }^{4,5}$ The theory of behavioral resilience states that a stressful experience can enhance the resilience of the individual to a subsequent stressor. ${ }^{4}$ The concept of resilience as being able to recover from stress and trauma is the same in all the described origins. Regardless of the origin and mechanism, these theories stand for the same concept of resilience and it is this knowledge that is of vital importance when assessing resilience in individuals.

The need for tools to measure mental resilience in adults has led to a range of different questionnaires., ${ }^{2,3,6}$ The Connor-Davidson Resilience Scale (CD-RISC) is such a questionnaire assessing the personal characteristics that embody resilience. However, the CD-RISC has a few disadvantages. One of these disadvantages is the absence of a reversed scoring system, which may lead to an increased risk of rating bias. To reduce this risk, the brief resilience scale (BRS) was developed, based on the CD-RISC, to which the reversed scored items were added. ${ }^{6}$ The BRS assesses the ability to bounce back from stressful situations and has proven to be negatively related to anxiety, depression, and negative effect.

It has been suggested that individuals who are considered resilient have a better mental and physical health through affective, cognitive, and behavioral coping pathways. ${ }^{7}$ It has been shown that happiness, which is positively correlated with resilience, has influence on the perception of health. ${ }^{8}$ In fact, Sabatini found that happiness was the best predictor of subjective health status. ${ }^{9}$ Identifying predictors of resilience, resilient characteristics, and processes that enhance resilience is pivotal to develop interventions to improve health outcomes.

Positive moods and confidence promote coping efforts and the pursuit of goals, leading to increased health and improved immune system functioning. ${ }^{7,10-12}$ The immune system is a topic of interest regarding mental resilience because it is thought that stress and depression exert influence on the immune system, and vice versa: resilience, negatively related to depression, is thought to correlate with a better functioning immune system. ${ }^{4,13,14}$

It is unclear to what extent individuals are aware of their objective immune status. However, individuals can describe and rate their perceived immune status. ${ }^{15}$ An example of such a perception is the experience of feeling unwell during a period of illness. This perception originates from the effects of certain interleukins and proinflammatory cytokines on the brain. ${ }^{16}$ These cytokines might induce behavioral changes, thought to be part of a motivational system altering behavior to facilitate recovery from infections. ${ }^{17}$ Through cytokine signaling, the objective immune status is linked to the perception of immune functioning and sickness. However, the scientific data available on the relationship between perceived immune functioning and mental resilience is still scarce. Therefore, the aim of this study is to examine the relationship between mental resilience and perceived health and immune status.

\section{Methods}

A survey was conducted among healthy young Dutch adults aged 18-30 years. Participants were recruited at the campus of Utrecht University, and most of them were students. Surveys were completed on location, and data were collected during spring 2016. Written informed consent was obtained from all participants; no formal ethics approval was required to conduct this research, according to the Central Committee on Research Involving Human Subjects (CCMO).

Mental resilience was assessed using the BRS. ${ }^{6}$ The BRS consists of six items that can be answered using a 5-point Likert scale ranging from strongly disagree to strongly agree. The average mental resilience score ranging from 0 to 6 was computed, with higher scores implying stronger mental resilience.

Perceived immune functioning and perceived health status were scored from 0 (very poor) to 10 (excellent), using single item questions. ${ }^{15}$ A yes/no question was asked to determine whether participants perceived reduced immune functioning at this moment. In addition, the immune function questionnaire (IFQ) was completed. ${ }^{18}$ The IFQ includes 19 items on weakened immune system functioning, such as sore throat, flu, cold sores, ear infection, and sudden high fever. The frequency of these immune-related illnesses $(0=$ never, $1=$ once or twice, $2=$ occasionally, $3=$ regularly, and $4=$ frequently) was also scored on a 5-point Likert scale. The overall IFQ score ranges from 0 to 76 , with higher scores implying worse immune functioning.

The BRS and IFQ have been used in several studies and reliability and validity have been demonstrated. ${ }^{6,18}$ The 1 -item scores of perceived immune functioning and health have been used successfully in previous research, and outcomes correlate well with IFQ scores. ${ }^{15}$

IBM SPSS statistics version 23 was used for data analysis. Independent samples $t$-tests and Pearson correlation tests were used to assess significant differences and correlations between various groups and variables. Fisher's $r$-to- $z$ 
transformation calculation was used to test for significant differences between correlations.

\section{Results}

Descriptive statistics of the test population are displayed in Table 1. A total of 779 individuals completed the survey. Their mean age was $21.4( \pm 2.7)$ years. Of these, $37.6 \%$ of the subjects were males and $62.4 \%$ of the subjects were females. The subject population was generally considered healthy, with a mean health score of a $7.5( \pm 1.0)$ and a perceived immune functioning score of $7.7( \pm 1.2)$. The mean overall IFQ score was $11.9( \pm 5.9)$. The average score of mental resilience was $3.4( \pm 0.7)$. Reduced perceived immunity was reported in only a minority of the subjects $(25.3 \%)$.

When correcting for gender, age, height, weight, smoker status, number of cigarettes smoked per week, alcohol consumption status, number of alcoholic drinks consumed per week, drug use, and frequency of past year drug use, mental resilience was significantly correlated with perceived health $(r=0.233, p=0.0001)$, perceived immune functioning ( $r=0.124, p=0.002)$, and the IFQ score $(r=-0.185, p=0.0001$; Figures 1 and 2).

Mental resilience scores were found to be significantly higher in men than in women ( $3.6 \pm 0.7$ vs. $3.3 \pm 0.7, p=0.001$ ). Males also graded their immune system significantly higher than females ( $7.9 \pm 1.2$ vs. $7.6 \pm 1.2, p=0.001)$. The mean \pm SD IFQ score was significantly lower in males (10.6 \pm 5.7 vs. $12.6 \pm 5.9, p<0.001)$ compared with females, suggesting a better functioning immune system in males. In addition,

Table I Demographics of the participants

\begin{tabular}{|c|c|c|c|}
\hline Measure & $\begin{array}{l}\text { Overall } \\
(n=779)\end{array}$ & $\begin{array}{l}\text { Men } \\
(n=286)\end{array}$ & $\begin{array}{l}\text { Women } \\
(n=473)\end{array}$ \\
\hline Age, years & $21.4( \pm 2.7)$ & $21.7( \pm 3.1)$ & $21.2( \pm 2.4)^{\mathrm{a}}$ \\
\hline Height, m & $1.76( \pm 0.10)$ & $1.84( \pm 0.07)$ & I.7I $( \pm 0.07)^{\mathrm{a}}$ \\
\hline Weight, kg & $68.7( \pm 11.6)$ & $75.6( \pm 11.8)$ & $64.3( \pm 8.9)^{\mathrm{a}}$ \\
\hline Perceived health status & $7.5( \pm 1.0)$ & $7.6( \pm 1.0)$ & $7.5( \pm 1.0)$ \\
\hline Perceived immune functioning & $7.7( \pm I .2)$ & $7.9( \pm 1.2)$ & $7.6( \pm 1.2)^{\mathrm{a}}$ \\
\hline Mental resilience score & $3.4( \pm 0.7)$ & $3.6( \pm 0.7)$ & $3.3( \pm 0.7)^{\mathrm{a}}$ \\
\hline IFQ score & $11.9( \pm 5.9)$ & $10.6( \pm 5.6)$ & $12.6( \pm 5.8)^{\mathrm{a}}$ \\
\hline Percentage of smokers, \% & 12.5 & 11.2 & 13.3 \\
\hline $\begin{array}{l}\text { Number of cigarettes } \\
\text { smoked/week }\end{array}$ & $0.7( \pm 2.4)$ & $0.8( \pm 2.8)$ & $0.6( \pm 2.2)$ \\
\hline $\begin{array}{l}\text { Percentage alcohol } \\
\text { consumers, } \%\end{array}$ & 81.2 & 82.6 & 79.9 \\
\hline $\begin{array}{l}\text { Number of alcoholic drinks } \\
\text { consumed/week }\end{array}$ & $6.2( \pm 9.3)$ & $8.4( \pm I \mid .8)$ & $4.7( \pm 6.8)^{\mathrm{a}}$ \\
\hline Percentage drug users, $\%$ & 26.8 & 32.4 & $23.2^{\mathrm{a}}$ \\
\hline $\begin{array}{l}\text { Frequency of past year } \\
\text { drug use }\end{array}$ & $7.0( \pm 35.7)$ & $9.0( \pm 38.0)$ & $6.0( \pm 34.9)$ \\
\hline
\end{tabular}

Notes: Mean (SD) values are shown. aSignificant differences $(p<0.05)$ between men and women.

Abbreviations: IFQ, immune function questionnaire; SD, standard deviation.

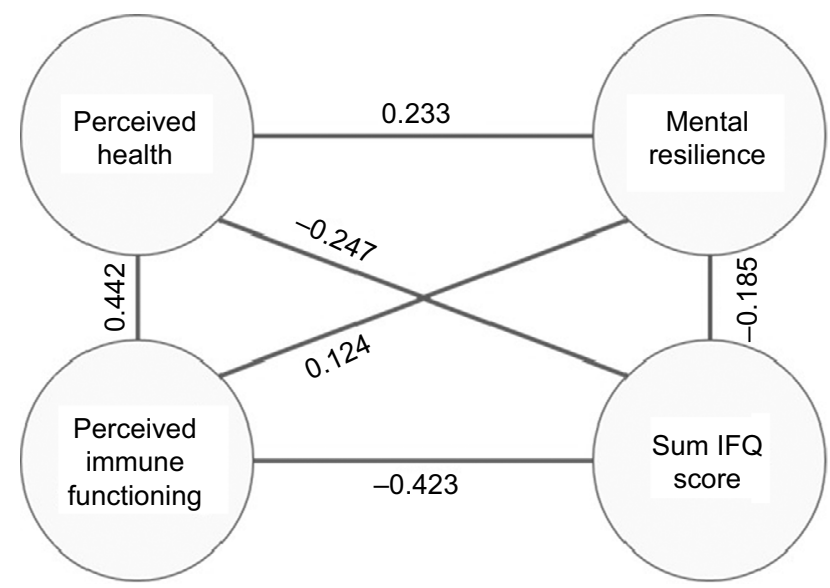

Figure I Summary of the associations between mental resilience, perceived immune functioning, and health.

Notes: Partial correlations are shown, corrected for gender, age, height, weight, smoker status, number of cigarettes smoked per week, alcohol consumption status, number of alcoholic drinks consumed per week, drug use, and frequency of past year drug use. All depicted correlations are statistically significant $(p<0.05)$.

Abbreviation: IFQ, immune function questionnaire.

significantly less men than women reported reduced perceived immune functioning ( $19.6 \%$ vs. $28.9 \%, p=0.005)$. No significant gender difference was found for perceived health. Also, the observed correlations between mental resilience and perceived health and immune status did not significantly differ between men and women.

Those who reported a reduced perceived immune status had significantly lower scores of perceived health $(7.1 \pm 1.0 \mathrm{vs}$. $7.6 \pm 0.9, p=0.001)$, perceived immune functioning $(7.0 \pm 1.2$ vs. $8.0 \pm 1.1, p=0.001)$, and mental resilience ( $3.2 \pm 0.7$ vs. $3.5 \pm 0.7, p=0.001)$ compared to participants reporting a healthy immune status. The mean IFQ score was significantly higher among people reporting a reduced perceived immune status $(14.9 \pm 6.2$ vs. $10.9 \pm 5.4, p=0.001)$. The observed correlations between mental resilience and perceived health and immune functioning did not significantly differ between those who reported a reduced perceived immune status and those who reported having a normal immune status.

\section{Discussion}

Albeit the observed associations between mental resilience and perceived health and immune status are modest, they support the notion that psychological well-being and mental strength has a positive impact on health and disease. Mental resilience scores were positively and significantly correlated with perceived health and perceived immune functioning. People who reported reduced immune functioning had significantly lower mental resilience scores compared with those who perceived their immune status as healthy. These results support the theory that resilient individuals have a 
A

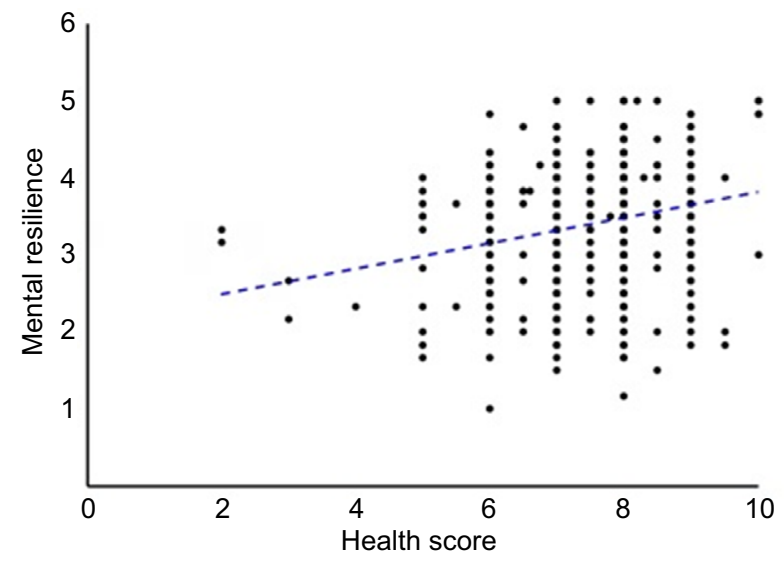

B

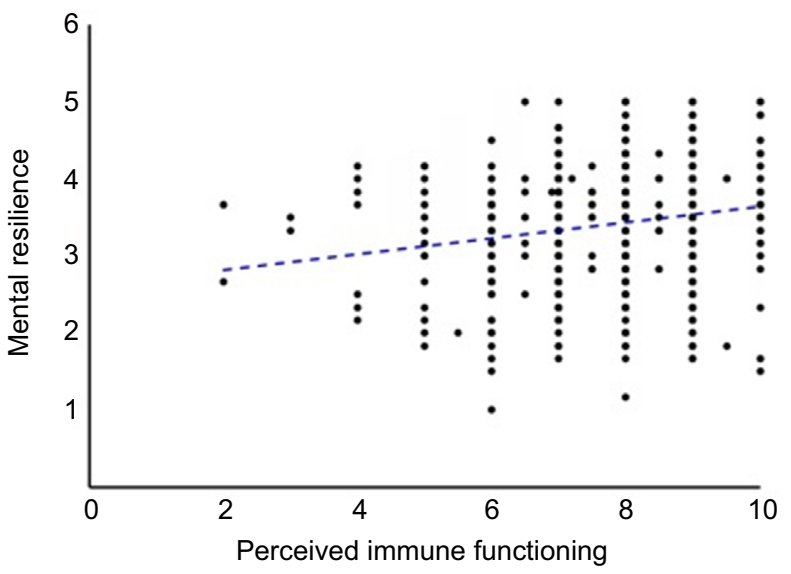

C

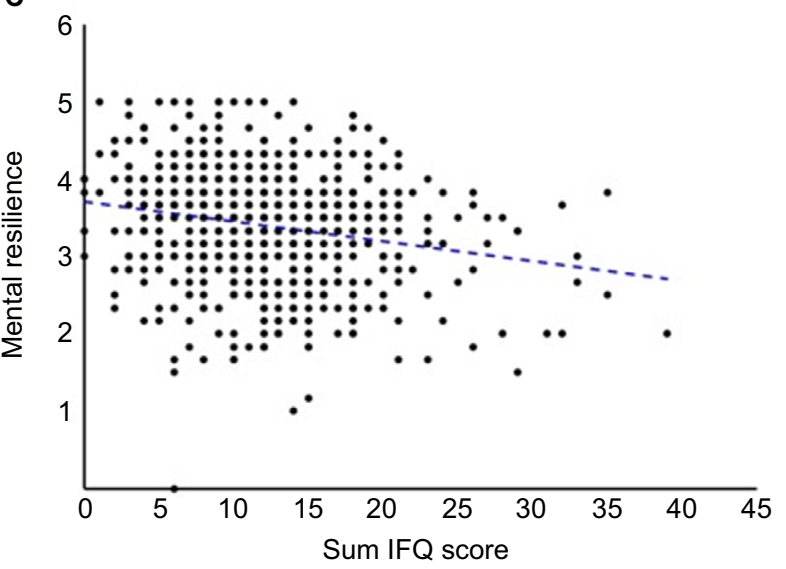

Figure 2 Correlations between mental resilience, health, and perceived immune functioning.

Notes: Individual scores of participants are shown, and the (uncorrected) correlation trendline for $(\mathbf{A})$ metal resilience and perceived health score $(r=0.228, p=0.000 \mathrm{I})$, (B) metal resilience and I-item perceived immune functioning $(r=0.175, p=0.000 \mathrm{I})$, and (C) mental resilience and the IFQ score $(r=-0.207$, $p=0.000 \mathrm{I})$. Correlations are significant if $p<0.05$.

Abbreviation: IFQ, immune function questionnaire.

better mental and physical health and better functioning immune system. ${ }^{4}$

A recent study among UK students revealed that resilient students were less likely to experience mental health difficulties and global subjective distress. Protective factors such as a strong family connection, high self-esteem, strong problem-solving skills, and peer support are characteristic for resilient students and contribute to experiencing less negative mental health outcomes. ${ }^{5}$ Given this, improvement of mental resilience may be a cost-effective way to improve general health and immune fitness. This can be achieved in various ways. For example, mindfulness sessions may improve their resilience status and help negotiate with life events and stress in both healthy people and patients. ${ }^{19}$

In this study, women had significant lower mental resilience scores than men. Our findings correspond with other research showing that women more often report lower selfconfidence and self-efficacy than men and claim to have less personal and material resources than men, which may result in a decreased sense of mastery among women. ${ }^{3}$
Also, women derive more happiness from social networks and family and are, therefore, more susceptible to relationship-oriented stressors than men. ${ }^{3,20}$ According to the "cost for caring" hypothesis, women gain stress when a stressful event occurs with a family member or a friend and, therefore, women have more adversities to overcome in daily life than men. All these factors cause women to experience more life events and to be more sensitive to them. ${ }^{3,20}$ Women also tend to cope with stressors in a different manner. Although men generally have an active approach to deal with stress, women often tend to ruminate and worry. It has been shown that the maladaptive manner in which women cope with stress lengthens depressive episodes, whereas the active approach of men is a protective factor for the illness causing effects of stressors. Another explanation for this phenomenon lies in traditional gender roles. ${ }^{21}$ Traditionally, men and women are socialized in distinct environments, where men are taught to be strong and independent and women to be passive, submissive, and dependent. This leads to the overrepresentation of women in low-paying jobs, where these traditional gender 
roles are reinforced while those with the highest levels of well-being are found among those employed in the highest employment grade. ${ }^{21}$

In this study, some remarks can be made. For example, our sample comprised significantly more women than men. This, however, reflects the Utrecht student population, and the sample size is large enough to reliably compare both genders. The participants were aged 18-30 years. It is, therefore, unclear if this young sample is representative for other age groups. Finally, more research is needed to directly compare perceived immune functioning with objective measurements of immune status. In this study, no saliva or blood samples were collected. In future studies, this should be implemented to also objectively assess the participants' immune status and allow a direct comparison with the participants' health perceptions.

Taken together, the findings of this study suggest a clear relationship between perceived health, perceived immune status, and mental resilience. That is, those with a better mental resilience score tend to have an improved health status and immune status compared to those with a worse mental resilience score.

\section{Acknowledgments}

An abstract of this paper was presented at the ECNP conference as poster with interim results. The abstract of the poster was published in European Neuropsychopharmacology, 2016, vol. 26: S367.

\section{Disclosure}

Joris C Verster has received grants/research support from the Dutch Ministry of Infrastructure and the Environment, Janssen Research and Development, Nutricia, Red Bull and Takeda, and has acted as a consultant for the Canadian Beverage Association, Central Bureau Drogisterijbedrijven, Coleman Frost, Danone, Deenox, Eisai, Janssen, Jazz, Purdue, Red Bull, Sanofi-Aventis, Sen-Jam Pharmaceutical, Sepracor, Takeda, Transcept, Trimbos Institute, and Vital Beverages. Aletta D Kraneveld has received grants/research support from Top Institute Pharma, NWO, Janssen, GSK, Nutricia Research, and Friesland Campina. Johan Garssen is a part-time employee of Nutricia Research. The other authors report no conflicts of interest in this work.

\section{References}

1. Shastri PC. Resilience: building immunity in psychiatry. Indian $J$ Psychiatry. 2013;55(3):224-234.

2. Ahern NR, Kiehl EM, Sole ML, Byers J. A review of instruments measuring resilience. Issues Compr Pediatr Nurs. 2009;29(2):103-125.

3. Hu T, Zhang D, Wang J. A meta-analysis of the trait resilience and mental health. Pers Indiv Differ. 2015;76:18-27.

4. Lewitus GM, Schwartz M. Behavioral immunization: immunity to selfantigens contributes to psychological stress resilience. Mol Psychiatry. 2009;14(5):532-536.

5. Lereya ST, Humphrey N, Patalay P, et al. The student resilience survey: psychometric validation and associations with mental health. Child Adolesc Psychiatry Ment Health. 2016;10:44.

6. Smith BW, Dalen J, Wiggins K, Tooley E, Christopher P, Bernard J. The brief resilience scale: assessing the ability to bounce back. Int J Behav Med. 2008;15(3):194-200.

7. Segerstrom SC, Castañeda JO, Spencer TE. Optimism effects on cellular immunity: testing the affective and persistence models. Pers Individ Differ. 2003;35:1615-1624.

8. Lü W, Wang Z, Liu Y, Zhang H. Resilience as a mediator between extraversion, neuroticism and happiness, PA and NA. Pers Individ Differ. 2014;63:128-133.

9. Sabatini F. The relationship between happiness and health: evidence from Italy. Soc Sci Med. 2014;114:178-187.

10. Stiller AL, Drugan RC, Hazi A, Kent S. Stress resilience and vulnerability: the association with rearing conditions, endocrine function, immunology, and anxious behavior. Psychoneuroendocrinology. 2011; 36(9):1383-1395.

11. Matzner P, Hazut $O$, Naim R, et al. Resilience of the immune system in healthy young students to 30 -hour sleep deprivation with psychological stress. Neuroimmunomodulation. 2013;20:194-204.

12. Sandvik AM, Bartone PT, Hystad SW, Philips TM, Thayer JF, Johnsen BH. Psychological hardiness predicts neuroimmunological responses to stress. Psychol Health Med. 2013;18(6):705-713.

13. Miller AH. Depression and immunity: a role for T cells? Brain, Behav Immun. 2010;24(1):1-8.

14. Lorenz T, van Anders S. Interactions of sexual activity, gender and depression with immunity. J Sex Med. 2014;11(4):966-979.

15. Donners AAMT, Tromp MDP, Garssen J, Roth T, Verster JC. Perceived immune status and sleep: a survey among Dutch students. Sleep Disord. 2015;2015:721607.

16. Dantzer R. Cytokine-induced sickness behaviour: a neuroimmune response to activation of innate immunity. Eur J Pharmacol. 2004; 500(1-3):399-411.

17. Dantzer R, Kelley KW. Twenty years of research on cytokine-induced sickness behaviour. Brain Behav Immun. 2007;21(2):153-160.

18. Reed P, Vile R, Osborne LA, Romano M, Truzoli R. Problematic internet usage and immune function. PLoS One. 2015;10(8): e 0134538.

19. Kinser P, Braun S, Deeb G, Carrico C, Dow A (2016). "Awareness is the first step": an interprofessional course on mindfulness and mindfulmovement for healthcare professionals and students. Complement Ther Clin Pract. 2016;25:18-25.

20. Boardman JD, Blalock CL, Button TMM. Sex differences in the heritability of resilience. Twin Res Hum Genet. 2008;11(1):12-27.

21. World Health Organization. Gender Discrepancies in Mental Health. Geneva: WHO. Available from: http://www.who.int/mental_health/ prevention/genderwomen/en/. Accessed September 6, 2016. 
The Journal of Multidisciplinary Healthcare is an international, peerreviewed open-access journal that aims to represent and publish research in healthcare areas delivered by practitioners of different disciplines. This includes studies and reviews conducted by multidisciplinary teams as well as research which evaluates the results or conduct of such teams or health care processes in general. The journal covers a very wide range of areas and welcomes submissions from practitioners at all levels, from all over the world. The manuscript management system is completely online and includes a very quick and fair peer-review system. Visit http://www.dovepress.com/ testimonials.php to read real quotes from published authors.

Submit your manuscript here: https://www.dovepress.com/journal-of-multidisciplinary-healthcare-journal 